\title{
INFLUENCIA DEL MODELO E IMAGEN DEL MÉDICO DURANTE LA FORMACIÓN DE PREGRADO EN UN GRUPO DE ESTUDIANTES DE MEDICINA DE LA UNIVERSIDAD DE ANTOFAGASTA
}

\author{
Cecilia Orellana Peña*, Mónica Rojas Urzúa**, Marianela Silva Zepeda***
}

\begin{abstract}
Resumen: Este trabajo tiene como objetivos conocer la influencia del modelo médico transmitido por quienes imparten docencia e identificar los valores de los estudiantes al ingresar a medicina, los valores transmitidos en pregrado y la importancia del modelo médico en esta etapa. Mediante una entrevista semiestructurada, previo consentimiento informado, se recopilaron percepciones de una cohorte de internos respecto de la imagen que tenían del médico al ingresar a medicina y su evolución durante la formación de pregrado. Se identifica un impacto tanto positivo como negativo de los docentes sobre los estudiantes. Se analiza esta influencia y se dan sugerencias concretas de cómo corregirla u optimizarla según el caso.
\end{abstract}

Palabras clave: bioética, ética clínica, educación médica, ética en docencia, valores

\section{INFLUENCE OF PHYSICIAN MODEL AND IMAGE DURING PREDEGREE TRAINING IN A GROUP OF MEDICAL STUDENTS AT ANTOFAGASTA UNIVERSITY}

\begin{abstract}
This study has as aims to know the influence of physician model transmitted by those who teach and to identify student's values when they initiate medical school, values transmitted before obtaining the degree and the importance of physician model in this stage. Through a semi structure interview, with previous informed consent, perceptions were collected from a cohort of students with respect to the image they had about physicians when entering medical school and its evolution throughout training. An impact both positive and negative was identified over students by professors. This influence is analyzed and concrete suggestions are made to correct or improve it in each case.
\end{abstract}

Key words: bioethics, clinical ethics, medical education, ethics in teaching, values

\section{INFLUÊNCIA DO MODELO E IMAGEM DO MÉDICO DURANTE A GRADUAÇÁO NUM GRUPO DE ESTUDANTES DE MEDICINA DA UNIVERSIDADE DE ANTOFAGASTA}

Resumo: Este trabalho tem como objetivos conhecer a influência do modelo médico transmitido pelos docentes e identificar os valores dos estudantes ao ingressar no curso médico, os valores transmitidos na graduação e a importância do modelo médico nesta etapa. Mediante uma entrevista semi-estruturada, com prévio consentimento informado, se recolheram percepções de uma coorte de internos sobre a imagem que tinham do médico ao ingressar no curso médico e sua evolução durante a sua graduação. Identifica-se um impacto tanto positivo como negativo dos docentes sobre os estudantes. Analisa-se esta influência e se oferecem sugestóes concretas de como corrigi-la ou otimizá-la de acordo com o caso.

Palavras-chave: bioética, ética clínica, educação médica, ética na docência, valores

\footnotetext{
* Nefróloga, Profesora Asistente Adjunta, Facultad de Medicina y Odontología, Departamento Ciencias Médicas, Universidad de Antofagasta, Chile Correspondencia: corellanap@gmail.com

** Matrona Servicio Neonatología, Hospital Regional Antofagasta. Académico Carrera de Medicina, Universidad de Antofagasta, Chile

*** Enfermera, Hospital Regional Antofagasta. Académico Asistente Adjunto, Facultad de Medicina y Odontología, Universidad de Antofagasta, Chile
} 


\section{Introducción}

La enseñanza de la medicina requiere de actividades prácticas en las que el tutor, un médico clínico, transmite conocimientos teóricos y destrezas propias de la profesión; sin embargo, también afloran actitudes del docente que en ocasiones pueden constituir un modelo negativo para los estudiantes. Centrado en entregar conocimientos y evaluar desempeño, el docente médico no siempre es consciente de cuán expuesto está a la observación y análisis de su actuar: es evaluado por los estudiantes en el día a día, en una serie de aspectos.

La enseñanza es un proceso social en el que ambas partes se perfeccionan mutuamente y en el cual la presentación de valores y disvalores es inseparable de la tarea docente. El profesionalismo demostrado por el docente constituirá un modelo que condicione la visión del estudiante respecto de la actividad para la que se prepara. El docente médico puede influir en la formación de actitudes de los estudiantes básicamente por tres caminos:

- Presentación de modelos de identificación: el estudiante se identifica con el modelo médico, pues "siempre quiso ser médico" y llega a la etapa de formación encontrándose con su modelo (lo que deseó ser toda su vida).

- Selección y valoración de la información que proporciona: el docente puede influir en el educando en su forma de ver la realidad. Cuando el estudiante alcanza la madurez para decidir lo bueno y lo malo en el actuar podrá hacer buen uso de lo entregado por el profesor. El profesor tiene la posibilidad de ser un facilitador del aprendizaje o un obstáculo.

- Empleo de los incentivos necesarios para el logro de un aprendizaje formativo: el modelo médico docente incentiva o penaliza las competencias mediante calificaciones.

El aprendizaje social adquirido en la relación docentealumno llega a ser más significativo que el cognitivo. La personalidad del modelo, su afectividad e implicación respecto del estudiante son factores mediadores del aprendizaje. La afectividad hacia el educando es clave en la pedagogía que intente ser eficaz: lleva a los estudiantes a ilusionarse con el aprender.

El propósito del presente estudio fue investigar cuánto y de qué forma influyen los docentes médicos en sus estudiantes, permitiendo desvelar posibles debilidades del modelo para después construir un perfil del docente médico que satisfaga los requerimientos del perfil profesional del egresado de medicina.

\section{Concepto de "educación"}

La educación debe aspirar a que su fin último sea la formación integral de la persona, entendida como un ser con necesidades, habilidades y potencialidades. La integralidad alude a que debe intervenir tanto en la dimensión cognitiva como en la axiológica (valores) y motora (habilidades y destrezas) de la persona.

Es indispensable considerar que los estudios de pregrado transcurren por lo general durante una etapa de la vida crucial para la madurez del individuo. Por otro lado, el estilo de aprendizaje -combinación de cuatro modalidades básicas: aprender con base en experiencias concretas, observación, conceptualización abstracta y experimentación activa- varía de una persona a otra. De ello deriva que la labor educativa tome en cuenta las condiciones que posibilitan la formación del individuo. ¿Cómo planifica el docente su estrategia para lograr el resultado esperado?

La respuesta es que toda estrategia docente debe fundamentarse en la dignidad de la persona (estudiante), puesto que la visión de todo proceso educativo es lograr el desarrollo de sus potencialidades bajo una visión global del individuo. Actualmente no es fácil ejercer la docencia en medicina. Estamos todavía inmersos en la era del conocimiento, en la cual el desafío para la educación implica un cambio profundo; hasta hace poco lo valórico y lo actitudinal eran trazas prácticamente imperceptibles en la formación de pregrado.

Recordemos que quienes imparten la docencia en las carreras de medicina son médicos de profesión, generalmente clínicos. En algunos casos, tienen un "gusto" por la docencia o han ganado un concurso para ocupar el cargo e ingresar a la universidad. No obstante, pareciera que estas características no son suficientes ni aseguran el éxito del proceso de enseñanza-aprendizaje. Aun cuando los médicos docentes carezcan de conocimientos de pedagogía, gravan sobre ellos algunas responsabilidades éticas por el hecho de participar en la enseñanza.

La ética formula juicios particulares de la conducta humana desde el punto de vista moral. Estos juicios se fundamentan en reglas que parten de principios, a su vez sustentados en las grandes teorías éticas. La docencia universitaria, por otra parte, es una manifes- 
tación de la conducta humana, por tanto, es objeto de un análisis ético. Entre las responsabilidades éticas del docente podemos mencionar: la obligación de enseñar, el compromiso con la verdad, con el alumno -varios de los principios de la bioética moderna a propósito de la relación médico-paciente tienen aplicación en la relación docente-alumno-, con la institución educativa, con la profesión médica y con la sociedad.

En la persona del docente médico convergen la ética de la docencia y la docencia de la ética, que en realidad no son sino diferentes expresiones del actuar de una misma persona. A ello se debe que no exista un día para hablar de ética, sino que cada día y cada acción vienen a estar impregnados de este concepto. Y así, por ejemplo, en la medida en que el docente respete la dignidad de su alumno, éste estará aprendiendo a respetar la dignidad de sus futuros pacientes. El modelo médico formativo deberá actuar dentro de un marco ético, estar identificado con los valores y principios morales que sustentan el sistema ético médico. Debe tener una idea muy clara de la estructura de su propio sistema de valores $y$ de la forma en que sus juicios personales influyen en las decisiones relacionadas con lo bueno o malo, así como también en sus alumnos, debiendo tener un conocimiento básico de la ética como disciplina. Sólo si el modelo médico hace propios estos preceptos y los incorpora a su jornada diaria estará formando futuros médicos integrales, con una sólida formación ética científica, y no solamente alumnos que se perfeccionan en áreas específicas de la medicina.

La Universidad de Antofagasta cuenta con el Hospital Clínico Regional de Antofagasta Dr. Leonardo Guzmán como su principal campo clínico para los estudiantes de pregrado de la carrera de medicina. Allí desarrollan actividades docentes tanto académicos del Departamento de Ciencias Médicas como médicos clínicos del Servicio de Salud de Antofagasta. Las actividades prácticas de los internos de $6^{\circ}$ y $7^{\circ}$ año de medicina son supervisadas en su mayoría por los médicos del Servicio de Salud en las diferentes unidades del hospital por las que rotan los internos. Los médicos de estos servicios desempeñan un rol docente-asistencial que lleva implícita una doble responsabilidad: el compromiso asumido con un grupo de alumnos al que se dedican diariamente, a la vez que responsabilidad de la atención de un número determinado de pacientes hospitalizados. Los médicos académicos de la universidad, en cambio, ejercen un rol exclusivamente docente, traducido en la atención diaria de un grupo de alumnos, visita a pacientes hospitalizados, análisis de sus patologías, posibilidad de asesorar al médico a cargo de esa sala, pero sin la responsabilidad del tratamiento específico ni pronóstico de ese enfermo.

El propósito de este estudio fue conocer la opinión de los estudiantes respecto del modelo de formación valórica que enfrentan, con la finalidad de formular mejoras en los ejes transversales del currículo. Se planteó como objetivo general: conocer la influencia que ejercen los modelos del profesional médico transmitidos por quienes imparten la docencia; como objetivos específicos: determinar los principales valores que poseen los estudiantes al inicio de la enseñanza de pregrado, identificar aquellos que se transmiten en el proceso de enseńanza de pregrado y señalar la importancia del modelo del profesional médico en la formación de pregrado en la carrera de Medicina.

\section{Metodología}

Se trató de un estudio exploratorio, transversal y cualitativo, basado en un paradigma interpretativo. Las investigadoras exploraron los discursos de los participantes, intentando determinar cómo ven ellos su realidad y experiencias a lo largo del tiempo.

El objeto del estudio fue abordado mediante una entrevista semiestructurada, previo consentimiento informado. Las temáticas recopilan percepciones con relación a la imagen del médico que tenían los estudiantes del pregrado antes de ingresar a la carrera de medicina y la evolución alcanzada por dicha imagen durante el proceso de formación universitaria. Las entrevistas fueron realizadas por un médico y una matrona durante los meses de octubre y noviembre de 2008. Se les explicó a los informantes que el objetivo de su participación era mejorar el modo como se realiza la docencia y que la información recogida se trataría en forma confidencial, respetando el anonimato.

Todos aceptaron participar. El formato de la entrevista semiestructurada consideró: edad, sexo, año de ingreso a la carrera y estudios universitarios previos. Se tomó nota de las respuestas a las siguientes preguntas:

1. ¿Cuál fue el principal motivo para decidirte a estudiar medicina?

2. La idea que tenías del médico al ingresar a la carrera, ¿se identifica con algún valor o característica? 
3. ¿Actualmente ha variado esa imagen? ¿En qué sentido?

4. ¿Puedes identificar algún(os) factor(es) que hayan influido en ese cambio?

5. En cuanto a tu futuro rol de médico, ¿qué imagen te gustaría proyectar? ¿Qué aspectos crees que tendrías que trabajar para lograrla?

\section{Análisis de datos y resultados}

Para analizar los datos se revisaron los discursos capturados, luego se ordenó la información reconociendo en las respuestas categorías que concentraban ideas similares de los informantes, lo que permitió agrupar la información para responder las preguntas del estudio.

Del paso anterior surgieron unidades temáticas que permitieron integrar la información y explicar la realidad estudiada. Para resguardar el anonimato de los informantes los discursos se señalan con letras A, $\mathrm{B}, \mathrm{C}, \mathrm{D}$, etc.

\section{Características de la población entrevistada}

La cohorte estaba compuesta por 20 mujeres y 32 varones, todos ellos se encontraban al término del $6^{\circ}$ año de la carrera. Se optó por internos de $6^{\circ}$ y no de $7^{\circ}$ año por considerar que la mayor o menor incertidumbre en relación con el futuro profesional podía influir en sus respuestas. Nos pareció que a fines de $6^{\circ}$ año podía haber mayor objetividad para responder. La edad promedio de los internos fue de 26 años (rango 23 a 31 años), con un promedio de 6 años cursando la carrera, aunque encontramos 6 personas que, al momento de la entrevista, llevaban 9 años. Sólo 13 de ellos (25\%) tienen algún familiar médico, en 5 casos se trata de un familiar directo (padre, madre, hermano). A eso se debe que la opinión de esta cohorte nos parece representativa de la opinión de cualquier miembro de la comunidad civil. En 20 casos estaba la experiencia de haber cursado previamente algún tiempo otra carrera universitaria, fundamentalmente del área de la salud.

\section{Principal motivo para estudiar la carrera de medicina}

Cuando se preguntó a los alumnos el principal motivo para escoger la carrera, destacó en sus respuestas idealismo, altruismo, solidaridad y vocación de servicio. Pocas se relacionaron con un punto de vista más personal, como el beneficio propio, la percepción fue "quise estudiar medicina para ayudar al prójimo”.

Una persona mencionó haberla elegido por un cierto desafío personal, dado el nivel de exigencia, y otra por la independencia laboral.

Aparentemente, el medio familiar no tuvo mayor influencia en la decisión de los alumnos.

A: “...carrera independiente... poder tomar decisiones y con ello ayudar a la gente..."

B: "... ayudar a las personas... servir a la gente desvalida..."

C: “...trabajar con personas... donde uno pudiera hacer un aporte..."

D: " ...yo siempre he tenido vocación de servicio... aportar a la salud pública..."

E: "...desde chico... el colegio da una impronta... retribuir de alguna forma a la sociedad..."

F: “...ser útil en la sociedad...”

G: "...querer ayudar a las personas...”

$\mathrm{H}$ : “...contacto con las personas... mezcla ciencia y humanismo..."

I: “...vocación en el camino... también una búsqueda religiosa..."

J: “...me gustaba el área biológica y ayudar a las personas..."

K: “...siempre me gustó, influencia de modelo familiar sin presión, mi padre es ginecólogo..."

L: “...por ser una carrera de gran exigencia, quería probarme y por la gran variedad de opciones de trabajo..."

M: "...por la independencia laboral, aunque ahora pienso que no es así...”

$\mathrm{N}$ : “...mejor manera de ser útil en la sociedad... el médico actúa en distintas situaciones con una opinión respaldada..."

\section{Identificación del médico con un valor o característica antes de ingresar a la carrera de medicina}

En relación con este punto, vuelve a aparecer el espíritu de servicio y expresiones altruistas prioritariamente, un poco más distanciada la capacidad intelectual, lo que llama la atención porque podría esperarse una mayor significación de este valor con relación a su quehacer futuro. 
Imagen del médico durante la formación de pregrado - Cecilia Orellana Peña, Mónica Rojas Urzúa, Marianela Silva Zepeda

\begin{tabular}{|l|c|}
\hline $\begin{array}{l}\text { Valor o } \\
\text { Característica }\end{array}$ & $\begin{array}{l}\text { No de apreciaciones percibidas por los } \\
\text { estudiantes respecto de la imagen del } \\
\text { médico }\end{array}$ \\
\hline Trabajador & 4 \\
\hline Honesto & 5 \\
\hline Responsable & 10 \\
\hline Capacidad intelectual & 16 \\
\hline Liderazgo & 10 \\
\hline Espíritu de servicio & 28 \\
\hline Empático & 10 \\
\hline Expresión altruista & 28 \\
\hline
\end{tabular}

F: “...despreocupación de algunos por los pacientes... me he decepcionado un poquito..."

G: "...ha cambiado para bien, ya no la veo tan inalcanzable, requiere harta dedicación, responsabilidad..."

H: “...sí ha cambiado, con la madurez mía... primero la veía como un profesional más, ahora es más amplio aún..."

I: “...sí ha cambiado un poco, no son muy empáticos, tampoco tan inteligentes, preocupados, responsables..."

3. Variación de la idea e imagen del médico que percibe el estudiante de pregrado en el $6^{\circ}$ año de la carrera de medicina

En general la imagen del médico ha cambiado. Si bien hay un proceso de maduración personal que influye en este aspecto, los discursos denotan cierta decepción, a la cual contribuye en gran medida el medio: estar inmerso en la realidad hospitalaria. Otro aspecto, en gran medida el objetivo de esta investigación, se vincula con el modelo de médico al que han estado expuestos durante estos ańos. Los alumnos perciben buenos y malos modelos, pero expresan que han aprendido a diferenciar y a extractar lo mejor de cada uno para incorporarlo a su acervo personal. Entre lo negativo seńalan un cierto mercantilismo, falta de compromiso con los pacientes y mayor preocupación por logros personales. Lamentan la falta de servicio público, empatía y responsabilidad entre sus modelos. Preocupa profundamente que mencionaran "algunos no cumplen normas éticas", son competitivos, les preocupan más sus logros que el paciente. Mencionan pocos aspectos positivos, pero rescatan que sí hay unos pocos modelos a seguir, humildes y comprometidos, pero son los menos. Estos sí son capaces de dar todo por el paciente y a veces llegan a comprometer con ello su vida personal y familiar.

A: “...sólo algunos tienen idea de médico ético dispuesto a dar todo por la vida del paciente".

B: "...he comprendido que el médico tiene mayor responsabilidad".

C: “...hay más diversidad de valores de lo que yo creía”.

D: “...la imagen del médico ideal no ha cambiado, en la práctica se ve que no todos son así...”

E: “...por lo que he visto, existen médicos muy preocupados y otros no".

J: “...sí ha cambiado, veo que no prima tanto el servicio público sino que lo económico, lo que me ha defraudado; tengo buenos tutores, pero no son empáticos y no dan buen trato a los pacientes..."

K: “...sí ha cambiado, hay médicos que cumplen el perfil, otros no cumplen normas éticas, son competitivos, se enfocan en logros médicos pero no en los pacientes, les interesa el reconocimiento..."

L: "...no ha cambiado, aunque he visto falta de compromiso..."

M: "...no ha cambiado, mi visión se ha humanizado..."

$\mathrm{N}$ : “...sí ha cambiado, uno se encuentra con cualquier tipo de persona con el "cartón de médico", tenía una imagen ideal como la que veo en mi padre, más noble, más preocupado..."

O: "...no ha cambiado, aunque hay médicos que no se portan a la altura de sus valores, no me aleja de los míos..."

P: “...sí ha cambiado, veo a los médicos más preocupados de sí mismos...”

\section{Factores que influyen en el cambio de imagen del médico durante el pregrado}

Entre los factores que influyeron en este cambio, mayoritariamente destacaron a los tutores y el medio: "estar dentro de un hospital y vivirlo por dentro"; asumir la realidad con los aspectos positivos y negativos del quehacer de los tutores en el día a día. Hay buenos modelos comprometidos, al punto de sacrificar su vida personal, y otros tantos que representan lo opuesto; son modelos en relación con lo que no harían o repetirían durante su ejercicio profesional. Los alumnos reconocen su condición de aprendices absorbiendo todo lo observado y vivido, pero con la capacidad de discriminar lo que realmente consideran un aprendizaje para su vida personal y profesional. 
A: “...tutores y el medio (campos clínicos)".

B: “... estar dentro de un hospital, vivirlo por dentro..."

C: "...interactuar con los docentes..."

D: "...he visto la realidad..."

E: "...ver desde dentro la carrera..."

F: "...médicos o tutores que he tenido han reforzado ese concepto..."

G: "...estar inmerso en el mundo médico..."

H: "...como todo proceso de formación, uno es como una esponja, recibe mucha información de todos lados... nace la capacidad de discriminar qué es lo bueno y qué es lo malo..."

I: "...es un arte la medicina y tiene que haber un maestro..."

J: “...cambia la visión teniéndolos tan cerca, uno aprende a valorarlos más..."

K: "...se van grabando mańas..."

L: "...depende de uno tomar lo bueno o lo malo, pero influye el tutor..."

M: “...hay médicos muy humildes y otros muy pedantes, hay cambios en el trato entre el sistema público y el privado..."

$\mathrm{N}$ : “...hay modelos que es inconveniente seguir por el impacto en la vida personal, son un ejemplo... nos muestran nuestro futuro... hay que rescatar lo positivo y no ser igual".

O: “...son modelos, si son negativos evitaremos seguirlos y viceversa... uno compara y rescata la forma de enfrentar situaciones..."

P: “...hay buenos modelos y otros que uno no desearía repetir..."

\section{Proyección de una imagen médica personal}

Cobran gran valor las respuestas a cómo se verían a futuro, qué aspectos les gustaría que se destacaran de su persona. La mitad de los internos consideró "ser empático" o "ponerse en el lugar del paciente"; posteriormente, "ser confiable, responsable y entregado". Menos importancia otorgaron a los conocimientos, honestidad y sencillez.

Llama la atención lo poco destacado que les pareció ser respetuoso y prudente, así como también la condición de líder y el ejercicio docente.

\begin{tabular}{|l|c|}
\hline $\begin{array}{l}\text { Valor o } \\
\text { característica }\end{array}$ & $\begin{array}{l}N^{\circ} \text { de apreciaciones percibidas por los estudiantes } \\
\text { respecto a la imagen médica personal }\end{array}$ \\
\hline Responsable & 12 \\
\hline Entregado & 19 \\
\hline Empático & 26 \\
\hline Honesto & 4 \\
\hline Confiable & 14 \\
\hline Conocimientos & 6 \\
\hline Sencillo & 4 \\
\hline Asertivo & 2 \\
\hline Autocrítico & 1 \\
\hline Respetuoso & 2 \\
\hline Prudente & 2 \\
\hline Líder & 1 \\
\hline Docente & 1 \\
\hline
\end{tabular}

\section{Discusión}

Sobre la base de los hallazgos, las autoras piensan que el modelo e imagen del médico como formador es fundamental en el proceso de aprendizaje de los futuros médicos. En la muestra estudiada se percibe un gran capital humano al ingreso a la carrera, denotando valores altruistas, vocación de servicio y una gran generosidad de parte de los estudiantes; sin embargo, el contacto con el medio y los tutores, más su propio proceso de maduración, hace que un gran número de ellos cambie su percepción. Se destaca el valor del modelo actitudinal en el proceso de formación de competencias de los estudiantes, el cual necesita ser optimizado para la mejora en el pregrado.

Se suele asociar la medicina con ciencias biológicas; sin embargo, lo que motivó a la mayoría de los entrevistados a optar por esta profesión fue el deseo de servir. También, cuando se reconoce una inclinación por la biología, ésta aparece asociada a servicio o dedicación a las personas. Si nos fijamos en las dos primeras acepciones de servir - "estar al servicio de alguien" y "estar sujeto a alguien por cualquier motivo haciendo lo que él quiere o dispone"-, apuntan a la virtud de la generosidad, voluntad de darse a los demás. Es decir, identificamos valores positivos concretos en quienes ingresan a esta carrera. Pensamos que nos encontramos ante un grupo humano generoso, cuyo norte al iniciar la carrera era realizarse profesionalmente ayudando a los demás.

Es cierto que estamos analizando una cohorte de 52 personas; no obstante, la inquietud social es en general un rasgo presente -y cada vez más- en los estudiantes de medicina(6). Identifican al profesional médico con 
valores, entre los cuales volvemos a encontrar generosidad: "capaz de darlo todo por el paciente", "integridad intachable", "profesión más noble", "uno cree que la mejor persona podría ser médico". Sería de interés que los docentes generaran instancias que les permitieran conocer las motivaciones y pensamientos de sus alumnos. Enfrentarse a este tipo de discursos no puede menos que mover a reconocer la responsabilidad de custodiar y cooperar en el desarrollo de esos valores.

Lo que motivó la investigación era saber si durante los años transcurridos desde el ingreso a la carrera se había deteriorado esa imagen un tanto idealizada del médico, que todos podemos haber tenido en mayor o menor medida al ingresar a la universidad. Los informantes fueron capaces de discernir lo que han visto durante los años cursados y el modelo de médico al que aspiran. En 34 entrevistados $(65,4 \%)$ esa imagen ha cambiado. Salvo dos internos que no identifican ningún factor, para los demás el detonante del cambio en esa imagen, tanto en sentido positivo como negativo, ha sido entrar en contacto con la realidad ("ver desde dentro la carrera”). Identifican los siguientes factores: contacto con los tutores, conocer el sistema hospitalario y, en tres casos, su maduración personal.

Nos parece que esta capacidad de discriminación refleja la madurez de los entrevistados. Muchos de los internos aclaran que su modelo de médico se mantiene, lo que han visto es que no todos los médicos que van conociendo lo encarnan. El discurso: “...veo que no prima tanto el servicio público sino que lo económico, lo que me ha defraudado; tengo buenos tutores pero no son empáticos y no dan buen trato a los pacientes...” puede constituir evidencia suficiente de la necesidad de que en todas las facultades de medicina se impartan nociones de ética y bioética. No nos referimos tan sólo a deontología médica; lo que defrauda es la falta de valores en el modelo.

Preguntamos dirigidamente si creían que la conducta de los médicos influía en los alumnos, para precisar si le asignaban la misma importancia que a los otros factores que ya habían mencionado. No esperábamos respuestas tan categóricas: 51 de los 52 entrevistados creían que sí había influido en ellos el comportamiento profesional de los médicos, y al responder lo hacían con expresiones como "evidente", "por supuesto", "absolutamente". Habíamos previsto que las respuestas pudieran ser un tanto "instintivas" y pedimos que asignaran un valor a esa influencia en escala de 1 a 10. El rango de respuestas es amplio pero la mayoría se concentra entre 6 y 9 . No obstante, no podemos ignorar a las cuatro personas que respondieron 10. ¿Se trata de personalidades más afectivas? ¿Son personas más vulnerables al ambiente en el que se desenvuelven? Son interrogantes para las que no tenemos respuesta, pero sí podríamos sugerir que las encuestas de satisfacción que se apliquen a los alumnos incluyan aspectos como éstos.

Revisamos la edad de los diez informantes que creen tener la madurez suficiente para discernir el buen del mal ejemplo y la edad promedio es de 26 años; no hay diferencia con el resto de los entrevistados. Al constatar cuánto tiempo han estado esas personas en la carrera, el promedio es de 6,6 años, ligeramente por encima del promedio general del grupo. Sin embargo, una persona lleva 9 años, dos llevan 8 y tres llevan 7 años. Esta mayor permanencia en el sistema podría explicar que al menos estos individuos hayan tenido tiempo de madurar y aprender a discernir, por supuesto que no es el mejor sistema.

Estos internos reconocen una influencia positiva en unos casos y negativa en otros: 21 entrevistados piensan que influye en ellos "para bien y para mal"; esta expresión, al igual que "se te pegan mañas", denota que aun cuando ellos no quieran adquirir malos hábitos se ven de algún modo indefensos. También preocupa la respuesta de una persona: "desanima y confunde". Cuatro entrevistados (7,7\%) reconocieron que la imagen previa del modelo médico había cambiado para bien.

El hecho de haber encontrado a 10 personas que creen tener la madurez necesaria para discernir qué modelo seguir es un aliciente, pero no nos excusa de la autoevaluación. Estos hallazgos confirman la necesidad de tener una actitud autocrítica con relación a cómo llevamos a cabo la enseñanza. Un modo de medir ese impacto podría ser a través de encuestas de evaluación docente apropiadas a las circunstancias y que incluyan aspectos actitudinales de los docentes.

Al preguntar por la imagen médica personal que querrían proyectar en un futuro próximo, queríamos indagar a qué aspectos de su formación dan mayor importancia los entrevistados: si a los "técnicos" o a los "valóricos". La mitad respondió "empático"; siguen en orden de frecuencia: confiable, responsable, entregado. Hubo quienes discriminaron entre espíritu de servicio y entrega, pero los agrupamos en un mismo índice temático por tratarse de sinónimos (entregar: ponerse 
en manos de alguien, sometiéndose a su dirección o arbitrio)(5).

Es interesante preguntarnos por qué estas respuestas a esta pregunta en concreto:

- ¿Les preocupa ser empáticos porque con ellos no lo han sido?

- ¿Esperan que los pacientes se sientan comprendidos por ellos, ya que ellos no lo fueron?

- ¿Será una advertencia de que entre los variados aspectos que abarca la formación de futuros médicos, debemos enseñar a comunicarse? En asignaturas como la tradicional "Semiología" nos preocupamos mucho de la información que deben recoger, pero sin atender al "cómo".

- Probablemente los programas de capacitación a los docentes en técnicas pedagógicas deban evaluar cómo se comunican, cómo conocen a sus alumnos y si saben escuchar.

Otro aspecto en cuyas causas valdría la pena profundizar es la poca significación atribuida a la condición de líder y al ejercicio docente.

Pese a la experiencia vivida, los integrantes de esta cohorte quieren encarnar el ideal de médico que tenían al ingresar a la universidad. Por lo general, les cuesta autoevaluarse. La última pregunta de la entrevista "iqué aspecto crees que tendrías que trabajar para lograr proyectar esa imagen?” pretendía indagar cuánto se conoce cada uno, si está consciente de sus fortalezas y de sus debilidades. Las respuestas más frecuentes fueron: estudio $(\mathrm{n}=14)$, comunicación $(\mathrm{n}=7)$, paciencia $(n=3)$. Nos parece relevante que 10 entrevistados no hayan sabido precisar qué aspectos deben trabajar para lograr su objetivo.

\section{Conclusiones}

1. Los tutores constituyen modelos valóricos tanto positivos como negativos. Al juzgar por las cifras, no son buenos transmisores de valores positivos.

2. La influencia ejercida por el modelo médico docente tiene un impacto considerable en el alumno y hay una proporción de estudiantes más vulnerable al modelo que no logra sustraerse a la influencia negativa.

3. Los estudiantes de pregrado son portadores de valores positivos al ingresar a la carrera de medicina. Corresponde a la facultad aportar los medios necesarios para custodiar y ser cauce para el crecimiento de esos valores.

4. Se detecta un subgrupo de la cohorte de mayor resiliencia o madurez ( $\mathrm{n}=18 ; 35 \%)$, en los cuales las experiencias vividas no han deteriorado su ideal de médico.

5. El actuar cotidiano del médico docente transmite al alumno que sus tutores constituyen una población heterogénea en cuanto a valores o motivación, y que se requiere menor capacidad intelectual que la que ellos imaginaban para esta carrera.

6. Discursos como: "...hay buenos modelos y otros que uno no desearía repetir..." deben llevarnos a pensar y reconocer que quien quiera dedicarse a la docencia debe estar dispuesto a dejarse evaluar y corregir, de modo que llegue a ser transmisor de valores positivos.

Basándose en los resultados obtenidos, las autoras proponen tres acciones concretas para ayudar a los médicos docentes a transmitir aquellos valores que un día les motivaron también a estudiar medicina:

1. Incorporar en la preparación docente que reciben los médicos clínicos conocimientos básicos de ética y bioética.

2. Hacer un diagnóstico de las debilidades actitudinales de los tutores, para luego aportar las herramientas que permitan establecer una relación docente-alumno adecuada y constituir un modelo apropiado.

3. En la selección de docentes, considerar una evaluación de las habilidades comunicacionales del candidato, tales como: capacidad para trabajar en equipo, dejarse corregir, saber escuchar.

\section{Bibliografía}

Aguerrondo I: "El nuevo paradigma de la educación para el siglo XXI". Disponible en: http://www.oei.es/administracion/ aguerrondo.htm

Bloch S.: Medical Students and Clinical Ethics. MJA 2003; 178: 167-169. 
Imagen del médico durante la formación de pregrado - Cecilia Orellana Peña, Mónica Rojas Urzúa, Marianela Silva Zepeda

Esteve JM. La docencia: competencias, valores y emociones. Disponible en: http:/www.oei.es/evp/PonenciaEsteve.pdf León F. Enseñar bioética: cómo transmitir conocimientos, actitudes y valores. Acta Bioethica 2008; 14(1): 11-18.

Lifshitz A. Ética en la docencia médica. Gac Méd Mex 2000; 136(4).

Manzanilla LM. La decisión de estudiar medicina. Segunda Asamblea de la Asociación Venezolana de Facultades de Medicina. Mérida (Venezuela), octubre de 1967.

Perales A. Ética y humanismo en la formación médica. Acta Bioethica 2008; 14(1): 30-37.

Real Academia de la Lengua. Diccionario de la Real Academia de la Lengua Española. Disponible en http://www.rae.es Rodríguez G, Gil J, García E. Metodología de la investigación cualitativa. Málaga: Aljibe; 1996.

Santibáñez M. Desafíos educativos para el profesor-médico. Reflexiones desde la educación, ARS Médica; 15(15) Disponible en: http://escuela.med.puc.cl/publ/arsmedica/Arsmedica15/Desafios.html

Suárez F, Díaz E. La formación ética de los estudiantes de medicina: La brecha entre el currículo formal y el currículo oculto. Acta Bioethica 2007; 13(1).

Recibido: 6 de julio de 2009

Aceptado: 25 de septiembre de 2009 